\title{
Consumption of whole grains and cereal fiber and total and cause-specific mortality: prospective analysis of 367,442 individuals
}

\author{
Tao Huang ${ }^{1}$, Min Xu' ${ }^{1}$ Albert Lee ${ }^{2}$, Susan Cho ${ }^{3}$ and Lu Qi $i^{1,4^{*}}$
}

\begin{abstract}
Background: Intakes of whole grains and cereal fiber have been inversely associated with the risk of chronic diseases; however, their relation with total and disease-specific mortality remain unclear. We aimed to prospectively assess the association of whole grains and cereal fiber intake with all causes and cause-specific mortality.

Methods: The study included 367,442 participants from the prospective NIH-AARP Diet and Health Study (enrolled in 1995 and followed through 2009). Participants with cancer, heart disease, stroke, diabetes, and self-reported end-stage renal disease at baseline were excluded.

Results: Over an average of 14 years of follow-up, a total of 46,067 deaths were documented. Consumption of whole grains were inversely associated with risk of all-cause mortality and death from cancer, cardiovascular disease (CVD), diabetes, respiratory disease, infections, and other causes. In multivariable models, as compared with individuals with the lowest intakes, those in the highest intake of whole grains had a 17\% (95\% Cl, 14-19\%) lower risk of all-cause mortality and $11-48 \%$ lower risk of disease-specific mortality (all $P$ for trend $<0.023$ ); those in the highest intake of cereal fiber had a 19\% (95\% Cl, 16-21\%) lower risk of all-cause mortality and 15-34\% lower risk of disease-specific mortality (all $P$ for trend $<0.005$ ). When cereal fiber was further adjusted, the associations of whole grains with death from CVD, respiratory disease and infections became not significant; the associations with allcause mortality and death from cancer and diabetes were attenuated but remained significant ( $P$ for trend <0.029).
\end{abstract}

Conclusions: Consumption of whole grains and cereal fiber was inversely associated with reduced total and cause-specific mortality. Our data suggest cereal fiber is one potentially protective component.

Keywords: Cereal fiber, Mortality, Whole grains

\section{Background}

Grains, also called cereals, are the seeds of plants cultivated for food. When whole, they include the germ, bran, and endosperm [1]. Most whole grains are abundant sources of dietary fiber and other nutrients, such as minerals and antioxidants, which have shown beneficial effects on human health including improvement of weight loss, insulin sensitivity, and lipid profile, as well as inhibition of systemic inflammation [2-4].

\footnotetext{
* Correspondence: nhlqi@channing.harvard.edu

'Department of Nutrition, Harvard School of Public Health, 665 Huntington

Ave, Boston, MA 02115, USA

${ }^{4}$ Channing Laboratory, Department of Medicine, Brigham and Women's

Hospital and Harvard Medical School, 75 Francis St, Boston, MA 02115, USA

Full list of author information is available at the end of the article
}

In epidemiology studies, evidence is accumulating indicating that consumption of whole grain products or their effective components, especially dietary fiber found in the grain, i.e., cereal fiber, may reduce the risk of chronic disease. Several recent meta-analyses taking into account a large number of subjects and prospective studies showed significant and consistent protective effects of high intake of whole grains and cereal fiber on type 2 diabetes [5], cardiovascular disease (CVD) [6], and certain cancers (e.g., colorectal cancer) [7]. In our earlier analysis in the Nurses' Health Study, we observed potential protective effects of whole grains on total or cardiovascular mortality in diabetic women [8]. Although the National Institutes of Health (NIH)-AARP Diet and Health Study previously reported that dietary 
fiber intake was inversely associated with the risk of total death and death from CVD, infectious diseases, and respiratory diseases [9], few studies have prospectively examined the associations of whole grains and its components, such as cereal fiber, with total or diseasespecific mortality.

In the present study, we used data from 367,442 people who were at risk for a total of 12.3 million personyears. We aimed to provide reliable estimates of independent associations between baseline whole grains and cereal fiber intake and the risk of total or cause-specific death from CVD, cancers, diabetes, and other diseases.

\section{Methods}

\section{Study population}

The NIH-AARP Diet and Health Study included 566,399 AARP members aged 50 to 71 from six US states (California, Florida, Louisiana, New Jersey, North Carolina, and Pennsylvania) and two metropolitan areas (Atlanta, Georgia, and Detroit, Michigan) [10]. Participants responded to a questionnaire mailed in October 1995 and December 1997. Details of the NIH-AARP Study have been previously described [11]. Among participants who returned the questionnaires with satisfactory dietary data, we excluded individuals who indicated that they were proxies for the intended respondent $(\mathrm{n}=15,760)$ as well as those who had cancer $(n=50,591)$, heart disease $(\mathrm{n}=80,254)$, stroke $(\mathrm{n}=12,812)$, diabetes $(\mathrm{n}=52,647)$, or self-reported end-stage renal disease at baseline $(\mathrm{n}=$ 1,299). We also excluded those who reported extreme consumption ( $>2$ times the interquartile ranges of BoxCox transformed intake) of total energy $(\mathrm{n}=3,771)$ and dietary fiber $(n=3,324)$. Exclusion of individuals reporting extreme energy intake is widely used in nutritional epidemiology studies since these participants are more likely to over- or under-report their intake [12]. After exclusions $(\mathrm{n}=198,957)$, the analytic cohort included 367,442 individuals. The NIH-AARP Diet and Health study was approved by the Special Studies Institutional Review Board of the US National Cancer Institute. All participants provided written informed consent.

\section{Assessment of dietary exposures}

At baseline, dietary intake was assessed with a selfadministered 124-item food frequency questionnaire (FFQ), which was an early version of the Diet History Questionnaire developed at the National Cancer Institute $[13,14]$. Participants were asked to report their usual frequency of intake and portion size over the past 12 months using 10 predefined frequency categories ranging from never to $6+$ times per day for beverages and from never to $2+$ times per day for solid foods with three portion size categories. The food items, portion sizes, and nutrient database were constructed using the US Department of Agriculture's 1994-1996 Continuing Survey of Food Intakes by Individuals. The FFQ used in the study was calibrated using two non-consecutive 24-hour dietary recalls in $1953 \mathrm{NIH-AARP}$ study participants. The nutrient database for dietary fiber was based on AOAC methods.

The whole grains were defined as the whole grain part of each product. The US Department of Agriculutre's Pyramid Servings Database enabled us to accurately estimate whole-grain intake from all foods in the FFQ. The sources of whole-grain intake in the FFQ used in our study were ready-to-eat cereals, high-fiber cereals, other fiber cereals, whole-grain breads or dinner rolls, cooked cereal, popcorn, pancakes, waffles, French toast or crepes, rice or other cooked grains, bagels, English muffins, tortillas, pasta, crackers, chips, cookies or brownies, sweet pastries, and pies. In this Continuing Survey of Food Intakes by Individuals dataset., whole grain foods were defined as those containing at least $25 \%$ whole grains and/ or bran. Main fibers are from fruit, grains, vegetables, and beans in the present study. Cereal fiber was defined as fiber from all cereals (e.g., ready-to-eat cereals, high-fiber cereals, cooked cereal, and other fiber cereals) and grainbased products.

In specifying numbers of deaths by intake quintile, the number of deaths is determined by the energyadjusted intake quintile for the entire population; when deaths are specific to a sex, we used the quintiles within the sex. We also collected demographic, anthropometric, and lifestyle information, including history of smoking (the number of cigarettes smoked per day), time of smoking cessation ( $<1$ years, 1 to 5 years, 5 to 10 years, or $\geq 10$ years before baseline), physical activity (never, rare, 1 to 2,3 to $4, \geq 5$ hours/week), alcohol intake (g/day) family history of cancers, menopausal hormone therapy use in women, and some medical conditions at baseline.

\section{Ascertaining mortality}

The AARP dataset denotes date of death and cause of death. There are 22 broad categories for cause of death. The modeling analysis for specific cause of death is performed with the study end date of 2008. For total mortality, models for study end dates in 2008 and 2009 were designed. Subjects with date of death after the study's end date are treated as alive at the end of the study, with no death or cause of death in the model. When the study end date was 2008, and there was a date of death but no cause of death for 2008 or earlier, the subject was not included in the modeling for cause of death, but only total mortality. Thus, when specifying numbers of deaths it depends on both the study end date and whether the cause of death field is missing. Vital status was determined through a periodic linkage of the cohort to 
the Social Security Administration Death Master File and follow-up searches of the National Death Index Plus for participants who matched the Social Security Administration Death Master File, cancer registry linkage, questionnaire responses, and responses to other mailings. The International Classification of Diseases, Ninth Revision [15] and the International Statistical Classification of Diseases, 10th Revision [16] were used to define death as follows: cancer (ICD-9, 140-239; ICD-10, C00-C97 and D00-D48), CVD (ICD-9, 390-398, 401-404, 410-429, and 440-448; ICD-10, I00-I13, I20-I51, and I70-I78), diabetes (ICD-9, 250; ICD-10, E10-E14), respiratory disease (ICD-9, 480-487 and 490-496; ICD-10, J10-J18 and J40-J47), infections (ICD-9, 001-139; ICD-10, A00-B99), and all other/unknown causes.

\section{Statistical analysis}

We used the Cox proportional hazards model to estimate hazard ratios (HRs) and two-sided 95\% confidence intervals (CIs) using the SAS PROC PHREG procedure (Version 9.1; SAS Institute Inc., Cary, NC, USA). Personyears of follow-up were calculated from the date of the baseline questionnaire until the date of death or the end of follow-up (December 31, 2009), whichever occurred first. Intake of whole grains and cereal fiber were adjusted for total energy intake using the residual method [17], and were categorized into quintiles.

We presented the results from four analysis models. Model 1, adjusted for age and gender; Model 2, adjusted for age, gender, the number of cigarettes smoked per day, and time of smoking cessation ( $<1$ years, 1 to 5 years, 5 to 10 years, or $\geq 10$ years before baseline); Model 3, adjusted for age, gender, the number of cigarettes smoked per day, time of smoking cessation ( $<1$ years, 1 to 5 years, 5 to 10 years, or $\geq 10$ years before baseline), race or ethnicity group, alcohol intake, education level, marital status (yes, no), health status (poor, fair, good, very good), obesity (underweight, overweight, obesity), physical activity, consumption of red meat (processed and fresh meat), total fruit and total vegetables, total energy intake, and hormone usage; and Model 4, based on Model 3 further adjusted for cereal fiber (whole grains analysis).

For missing data in each covariate, we created indicator variables. Overall, missing data was less than $5 \%$. The model results summary includes the results of statistical tests for trend in the response for the risk variable. Quintiles Trend $P$ denotes the $P$ value when the median value within the risk variable quintile is included in the hazard model as linear.

\section{Results}

Table 1 shows baseline characteristics of study participants $(n=367,442)$, according to intake of whole grains and cereal fiber. During an average of 14 years of follow-up (total person-years, 5,148,760), we documented 46,067 deaths, among them 11,283 from CVD, 19,043 from cancer, 371 from diabetes, 3,796 from respiratory disease, 922 from infection, and 5,223 from other causes. At baseline, intakes of whole grains and cereal fiber were inversely correlated with prevalence of overweight, obesity, and current smoking, as well as intake of red meat. The levels of moderate and vigorous physical activity were higher among participants with higher intakes of whole grains or cereal fiber than those with lower intakes.

\section{Whole grains and cereal fiber intake with total mortality}

In age- and gender-adjusted analysis (Model 1), we found that intake of whole grains were inversely associated with all-cause mortality (Table 2). As compared with the lowest quintile, the HRs across increasing quintiles of whole grain intake were 0.78 (95\% CI, 0.76-0.80), 0.70 (95\% CI, 0.68-0.72), 0.63 (95\% CI, 0.61-0.65), and 0.61 (95\% CI, $0.59-0.62)(P$ trend $<0.0001)$. Further adjustment for smoking status and time since smoking cessation (Model 2) did not appreciably change the associations. When the models further included race/ethnicity, education, marital status, self-rated health status, obesity (underweight, overweight, and obesity), physical activity, use of menopausal hormone therapy, and intake of alcohol, red meat, fruits, vegetables, and total energy (Model 3), the highest quintile of whole grain intake was associated with 17\% (95\% CI, 14-19\%) lower risk of all-cause mortality $(P$ trend $<0.0001)$. The associations between whole grain intake and all-cause mortality was attenuated, the highest quintile of whole grain intake was associated with $6 \%$ (95\% CI, 3-10\%) lower risk, but remained significant when cereal fiber was additionally adjusted (Model 4; $P$ trend $=0.002$ ). These results suggested that the protective effects of whole grain may be due, at least in the main part, to its cereal fiber component.

Similarly, we found that cereal fiber intake was significantly associated with all-cause mortality in ageand gender-adjusted and multivariate-adjusted models (Models 1, 2 and 3; all $P$ trend $<0.0001$; Table 3). In model 3 , the highest quintile of cereal intake was associated with $19 \%(16-21 \%)$ lower risk of all-cause mortality $(P$ trend $<0.0001)$.

\section{Whole grains and cereal fiber intake with cause-specific mortality}

We next tested the associations for cause-specific mortalities. In age- and gender-adjusted and multivariate adjusted models (Models 1, 2 and 3), intakes of whole grains or cereal fiber were inversely associated with risk of death from CVD, cancer, diabetes, respiratory disease, infections, and other/unknown causes (all $P$ trend $<0.023$ ). In Model 3, as compared with the lowest quintiles, people in the highest quintile of whole grain intake had $11 \%$ 
Table 1 Baseline characteristics of the study participants according to intake of whole grains and cereal fiber

\begin{tabular}{|c|c|c|c|c|c|c|c|}
\hline & \multirow{2}{*}{$\begin{array}{l}\text { Total } \\
\text { participants }\end{array}$} & \multicolumn{3}{|c|}{ Whole grains } & \multicolumn{3}{|c|}{ Cereal fiber } \\
\hline & & $\overline{\mathrm{Q1}}$ & Q3 & Q5 & $\overline{\mathrm{Q1}}$ & Q3 & Q5 \\
\hline$n$ & 367,442 & 73,488 & 73,489 & 73,489 & 73,488 & 73,489 & 73,489 \\
\hline Age, mean years & 61.7 & 61.1 & 61.7 & 62.1 & 61.0 & 61.7 & 62.2 \\
\hline Female, \% & 43.9 & 30.7 & 51.9 & 40.4 & 35.0 & 49.9 & 40.2 \\
\hline Whites, \% & 92.9 & 92.6 & 92.9 & 93.2 & 91.2 & 93.0 & 94.4 \\
\hline College graduate, $\%$ & 41.0 & 35.2 & 41.4 & 44.9 & 36.2 & 40.4 & 46.4 \\
\hline Married, \% & 68.1 & 72.7 & 65.2 & 68.9 & 69.8 & 66.1 & 69.9 \\
\hline Moderate physical activity (3-4 times/week), \% & 27.3 & 23.0 & 27.8 & 30.6 & 23.6 & 27.2 & 30.9 \\
\hline Vigorous physical activity ( $\geq 5$ times/week), $\%$ & 19.2 & 17.5 & 18.0 & 23.6 & 18.1 & 17.6 & 24.1 \\
\hline Overweight, \% & 42.4 & 44.5 & 42.0 & 41.0 & 43.9 & 42.2 & 41.1 \\
\hline Obesity, \% & 19.6 & 22.7 & 19.7 & 16.6 & 23.6 & 20.0 & 15.0 \\
\hline Very good or excellent self-report health, \% & 61.4 & 56.5 & 62.2 & 64.3 & 58.8 & 61.4 & 65.0 \\
\hline Previous or current use of postmenopausal hormone therapy, \% & 55.0 & 47.9 & 55.6 & 58.1 & 45.5 & 54.4 & 59.2 \\
\hline Former smoker, \% & 48.7 & 48.5 & 48.2 & 49.5 & 47.6 & 48.4 & 50.2 \\
\hline Current smoker, \% & 12.8 & 21.4 & 11.3 & 8.0 & 21.4 & 11.5 & 7.0 \\
\hline Median total energy intake (kcal/d) & 1,805 & 2,394 & 1,527 & 1,855 & 2,330 & 1,563 & 1,832 \\
\hline Median alcohol intake (g/d) & 14.7 & 32.5 & 10.0 & 8.8 & 27.5 & 11.6 & 9.9 \\
\hline \multicolumn{8}{|l|}{ Median servings of food } \\
\hline Red meat (oz/d) & 2.0 & 3.1 & 1.6 & 1.7 & 3.1 & 1.71 & 1.6 \\
\hline Fruits (cup eq/d) & 2.0 & 2.1 & 1.8 & 2.2 & 2.3 & 1.8 & 2.2 \\
\hline Vegetables (cup eq/d) & 1.9 & 2.2 & 1.7 & 2.0 & 2.3 & 1.7 & 2.0 \\
\hline
\end{tabular}

(respiratory disease) to $48 \%$ (diabetes) lower risk of causespecific mortality, while people in the highest quintile of cereal fiber intake had $15 \%$ (cancer) to $34 \%$ (diabetes) lower risk of cause-specific mortality.

When cereal fiber was further adjusted, the associations of whole grains with death from CVD, respiratory disease, infections, and other causes became non-significant; however, the associations with death from cancer and diabetes remained significant $(P$ trend $<0.029)$.

\section{Discussion}

In this large prospective cohort study of the US population, we found that high consumption of whole grains or cereal fiber was significantly associated with reduced risk of all-cause mortality and death from CVD, cancer, diabetes, respiratory disease, infections, and other causes. As compared with individuals with the lowest intake of whole grains, those in the highest intake group had a $17 \%$ lower risk of all-cause mortality and 11 to $48 \%$ lower risk of disease-specific mortality. As compared with individuals with the lowest intake of cereal fiber, those in the highest intake group had a 19\% lower risk of all-cause mortality and 15 to $34 \%$ lower risk of disease-specific mortality. Furthermore, our results suggested that the protective effects of whole grains may due, at least in the main part, to its cereal fiber component.
To the best of our knowledge, the present study is, thus far, the largest in size regarding deaths in a prospective setting. Our findings are concordant with previously observed protective effects of whole grain intake on CVD, diabetes, and certain cancers $[18,19]$. Based on a metaanalysis of six cohort studies including 286,125 participants and 10,944 cases, a two servings per day increment in whole grain consumption was associated with a $21 \%$ (95\% CI, 13-28\%) decrease in risk of type 2 diabetes after adjustment for potential confounders and BMI [5]. These findings were confirmed by Ye et al.'s meta-analysis [20], in which it was also reported that compared with never/ rare consumers of whole grains, individuals consuming 48 to $80 \mathrm{~g}$ of whole grains per day (3 to 5 serving/day) had a $21 \%$ lower risk of CVD (relative risk $=0.79$; 95\% CI, 0.74-0.85). Inverse associations were also reported between intake of whole grains and incident hypertension [21]. In a meta-analysis of 25 prospective studies, the summary relative risk of developing colorectal cancer for $10 \mathrm{~g}$ daily of cereal fiber was 0.90 (95\% CI, 0.83-0.97), pooled results from six studies showed the relative risk for an increment of three servings daily of whole grains was 0.83 (95\% CI, 0.78-0.89) [7]. High whole grain intakes have been related to reduced risk of other cancers, such as digestive cancer, in prospective studies, although the protective effects were not consistently observed [22,23]. 
Table 2 Association of whole grain intake with total and cause-specific mortality

\begin{tabular}{|c|c|c|c|c|c|c|}
\hline \multirow{2}{*}{$\begin{array}{l}\text { All } \\
\text { participants }\end{array}$} & \multicolumn{5}{|c|}{ Whole grains (oz eq/d) } & \multirow[t]{3}{*}{$P$ trend } \\
\hline & Q1 $(n=41,248)$ & Q2 $(n=41,248)$ & Q3 $(n=41,249)$ & $\mathrm{Q} 4(\mathrm{n}=41,248)$ & Q5 $(n=41,249)$ & \\
\hline & 0.13 & 0.30 & 0.47 & 0.69 & 1.20 & \\
\hline
\end{tabular}

\section{Causes of death}

\section{All cause}

No. of deaths

Model 1

Model 2

Model 3

Model 4

\section{Cardiovascular disease}

No of deaths

Model 1

Model 2

Model 3

Model 4

\section{Cancer}

No. of deaths

Model 1

Model 2

Model 3

Model 4

\section{Diabetes}

No. of deaths

Model 1

Model 2

Model 3

Model 4

\section{Respiratory disease}

No. of deaths

Model 1

Model 2

Model 3

Model 4

\section{Infections}

No. of deaths

Model 1

Model 2

Model 3

Model 4
$3,796 \quad 1,123$

46,067

11,845
1.00
1.00
1.00
1.00

11,283

2,921

1.00

1.00

1.00

1.00

19,043

4,836
1.00
1.00
1.00
1.00

371

113

1.00

1.00

1.00

1.00

1.00

1.00

1.00

1.00

922

251

1.00

1.00

1.00

1.00
9,450

$0.78(0.76-0.80)$

$0.88(0.86-0.91)$

$0.93(0.90-0.95)$

$0.96(0.93-0.99)$

2,330

$0.78(0.74-0.83)$

$0.88(0.83-0.93)$

$0.93(0.88-0.98)$

$0.96(0.91-1.02)$

3,912

$0.79(0.76-0.83)$

$0.91(0.87-0.95)$

$0.94(0.90-0.98)$

$0.96(0.92-1.00)$

72

$0.62(0.46-0.84)$

$0.67(0.49-0.90)$

$0.71(0.53-0.96)$

$0.74(0.54-1.00)$

802

$0.69(0.63-0.75)$

$0.88(0.80-0.96)$

0.99 (0.90-1.09)

$1.02(0.93-1.12)$

3,616

$0.71(0.68-0.75)$

$0.86(0.83-0.90)$

$0.91(0.87-0.95)$

$0.95(0.90-0.99)$

73

$0.62(0.46-0.83)$

$0.67(0.50-0.91)$

$0.76(0.56-1.03)$

$0.81(0.59-1.13)$

673

$0.56(0.50-0.61)$

$0.79(0.72-0.87)$

$0.94(0.85-1.03)$

$1.00(0.90-1.11)$

184

$0.71(0.59-0.86)$

163

$0.61(0.50-0.75)$

$0.69(0.57-0.84)$

$0.78(0.64-0.96)$

$0.84(0.70-1.02)$

$0.87(0.71-1.06)$

$0.83(0.67-1.04)$
8,054

$0.63(0.61-0.65)$

$0.78(0.75-0.80)$

$0.85(0.82-0.87)$

$0.92(0.89-0.96)$

1,914

$0.60(0.57-0.64)$

$0.73(0.69-0.77)$

$0.81(0.77-0.86)$

$0.90(0.84-0.97)$

3,388

0.65 (0.62-0.68)

$0.82(0.79-0.86)$

$0.88(0.84-0.92)$

$0.93(0.88-0.98)$

66

$0.54(0.40-0.73)$

$0.60(0.44-0.82)$

$0.72(0.53-0.99)$

$0.78(0.55-1.13)$

606

$0.48(0.43-0.53)$

$0.74(0.67-0.82)$

$0.91(0.82-1.01)$

$1.01(0.90-1.14)$
161

$0.58(0.48-0.71)$

$0.68(0.55-0.83)$

$0.79(0.65-0.97)$

$0.87(0.69-1.10)$
8,024

$0.61(0.59-0.62)$

$<0.0001$

$0.77(0.75-0.79)$

$<0.0001$

$0.83(0.81-0.86)$

$<0.0001$

$0.94(0.90-0.97)$

0.002

1,997

$<0.0001$

$0.60(0.57-0.64)$

$<0.0001$

$0.75(0.71-0.80)$

$<0.0001$

$0.83(0.78-0.88)$

$<0.0001$

$0.95(0.88-1.03) \quad 0.188$

3,291

$0.61(0.59-0.64)$

$<0.0001$

$0.80(0.76-0.84)$

$<0.0001$

$0.85(0.81-0.89)$

$<0.0001$

$0.93(0.88-0.99)$

0.025

47

$0.37(0.27-0.52)$

$<0.0001$

$0.42(0.30-0.60)$

$<0.0001$

$0.52(0.37-0.75)$

0.0009

$0.57(0.37-0.89) \quad 0.029$

592

$0.45(0.41-0.50)<0.0001$

$0.74(0.67-0.82)<0.0001$

$0.89(0.80-0.98) \quad 0.0099$

$1.03(0.91-1.18) \quad 0.67$

163

$0.57(0.47-0.70)<0.0001$

$0.68(0.55-0.83) \quad 0.0002$

$0.77(0.62-0.95) \quad 0.02$

$0.89(0.68-1.16) \quad 0.54$ 
Table 2 Association of whole grain intake with total and cause-specific mortality (Continued)

\begin{tabular}{|c|c|c|c|c|c|c|c|}
\hline \multicolumn{8}{|c|}{ Other/unknown causes } \\
\hline No. of deaths & 5,223 & 1,206 & 1,058 & 1,038 & 940 & 981 & \\
\hline Model 1 & & 1.00 & $0.86(0.79-0.93)$ & $0.82(0.76-0.90)$ & $0.71(0.66-0.78)$ & $0.72(0.66-0.78)$ & $<0.0001$ \\
\hline Model 2 & & 1.00 & $0.91(0.84-0.99)$ & $0.90(0.83-0.98)$ & $0.80(0.73-0.87)$ & $0.81(0.74-0.88)$ & $<0.0001$ \\
\hline Model 3 & & 1.00 & $0.97(0.88-1.06)$ & $0.97(0.89-1.06)$ & $0.87(0.79-0.96)$ & $0.86(0.78-0.94)$ & 0.0001 \\
\hline Model 4 & & 1.00 & $0.99(0.91-1.09)$ & $1.03(0.93-1.13)$ & $0.96(0.86-1.06)$ & $0.98(0.87-1.09)$ & 0.54 \\
\hline
\end{tabular}

Data are hazard ratios (HR) and 95\% confidence interval (Cl). The numbers of deaths are for participants who died during follow-up. The co-variables are baseline assessments.

Model 1, Adjusted for age and gender; Model 2, Adjusted for age, gender, the number of cigarettes smoked per day, and time of smoking cessation (<1 years, 1 to 5 years, 5 to 10 years, or $\geq 10$ years before baseline); Model 3, Adjusted for age, gender, the number of cigarettes smoked per day, time of smoking cessation ( $<1$ years, 1 to 5 years, 5 to 10 years, or $\geq 10$ years before baseline), race or ethnicity group, alcohol intake, education level, marital status (yes, no), health status (poor, fair, good, very good), obesity (underweight, overweight, obesity), physical activity, consumption of red meat, total fruit and total vegetables, total energy intake, and hormone usage. Model 4, Based on Model 3 further adjusted for cereal fiber.

Very few previous studies have examined the relationship between whole grains and their components with mortality in humans. Our findings are consistent with the results reported in the Nurses' Health Study, in which whole grain intake, especially bran, was associated with lower all-cause and CVD mortality in diabetic women [24]. Similarly, higher fiber intake was associated with lower total mortality, particularly mortality from circulatory, digestive, and non-CVD non-cancer inflammatory diseases in a large European prospective study of 452,717 men and women [25]. In a previous analysis among our study samples, it was found that intake of fiber from grains but not from other sources was inversely related to all-cause mortality and death from cancer, CVD, infections, and respiratory disease [9]. In this updated analysis, we found cereal fiber intake was inversely associated with death from diabetes. However, we did not report the associations of specific types of whole grain foods/products with mortality and cause-specific mortality, since it is hard to further differentiate such food groups; this presents a limitation of this observational study.

In addition, we found that the associations of whole grains with death from CVD, respiratory disease, and infections became non-significant after adjustment for cereal fiber intake. The associations with total mortality and death from cancer and diabetes were also largely attenuated, although they remained significant after adjustment for cereal fiber intake. These observations suggest that the protective effects of whole grains on mortality are at least partly mediated by its cereal fiber component. Such a postulation is supported by previous evidence that shows cereal fiber intake is related to an improvement of insulin sensitivity and lipid profile, an increase in protective molecules such as adiponectin, and a reduction in inflammation markers [26-28].

The protective effect of whole grains and fiber consumption on risk of mortality is biologically plausible. Dietary fiber intake is associated with lower levels of inflammation markers, such as C-reactive protein, and tumor necrosis factor $\alpha$ receptor 2 , which play key roles in chronic inflammatory conditions $[29,30]$. Whole grain foods are rich in fiber. Therefore, the anti-inflammatory effects of dietary fiber may help explain, at least in part, the inverse associations of whole grains and fiber consumption with chronic disease death. Moreover, whole grains and cereal fiber have a high content of antioxidants, vitamins, trace minerals, phenolic acids, lignans, and phytoestrogens, which have been associated with a reduced risk of colorectal cancer [31] and lower risk of death from non-cardiovascular, non-cancer inflammatory diseases and respiratory system diseases [32]. In addition, dietary fibers have specific and unique impacts on intestinal microbiota composition and metabolism $[33,34]$. Additionally, recent studies have related gut microbiota with various chronic diseases such as obesity, CVD, diabetes, and cancer [34,35]. Further functional investigations are warranted to verify these potential mechanisms.

\section{Strengths and limitations of the study}

In our study cohort, both whole grains and cereal fiber were correlated with high levels of physical activity and better health status, as well as with low BMI, low levels of smoking, and low intakes of alcohol and red meat. However, our results were less likely due to the potential confounding of these factors because careful adjustment for these factors in our analyses did not significantly change the results. Nevertheless, we acknowledge that the positive associations may still be related to residual confounding of non-measured covariates. Reverse causality might also affect the associations, since people with chronic disease might modify their eating habits by consuming healthy foods including those rich in whole grains and cereal fiber. In our analyses, however, we have excluded patients with cancer, heart disease, and diabetes at baseline and only analyzed the associations with incident cases. Whole grains and cereal fiber intakes were evaluated by self-report at a single time point. It is 
Table 3 Association of cereal fiber intake with total and cause-specific mortality

\section{All participants \\ Q1 $(n=73,488)$}

2.02

\section{Causes of death}

\section{All cause}

No. of death

Model 1

46,067

Model 2

Model 3

\section{Cardiovascular disease}

11,283

No. of deaths

Model 1

Model 2

Model 3

\section{Cancer}

No. of deaths

19,043

Model 1

Model 2

Model 3

Diabetes

No. of death

371

Model 1

Model 2

Model 3

\section{Respiratory disease}

No. of deaths

Model 1

3,796

Model 2

Model 3

Infections

No. of deaths

922

230

Model 1

Model 2

Model 3

\section{Other/unknown causes}

No. of deaths

5,223

Model 1

Model 2

Model 3

Cereal fiber $(g / d)$

Q2 ( $n=73,488)$

4.15

5.27

11,700

1.00

1.00

2,901

1.00

1,082

1.00

9,652

$0.80(0.78-0.83)$

$0.89(0.87-0.92)$

$0.93(0.90-0.95)$

2,368

$0.80(0.76-0.85)$

$0.88(0.83-0.93)$

$0.93(0.87-0.98)$

3,974

$0.81(0.78-0.85)$

$0.91(0.87-0.95)$

$0.94(0.90-0.98)$

8,664

$0.70(0.68-0.72)$

$0.83(0.81-0.85)$

$0.87(0.85-0.90)$

2,094

$0.69(0.65-0.73)$

$0.80(0.76-0.85)$

$0.86(0.81-0.91)$

3,616

$0.72(0.69-0.76)$

$0.87(0.83-0.91)$

$0.90(0.86-0.95)$

92

$0.92(0.69-1.22)$

72

$0.97(0.73-1.29)$

$0.70(0.52-0.95)$

$0.77(0.56-1.04)$

$1.00(0.74-1.35)$

$0.83(0.60-1.15)$

866

$0.75(0.68-0.82)$

$0.91(0.83-1.00)$

652

0.54 (0.49-0.59)

$0.76(0.69-0.84)$

$0.82(0.74-0.91)$

$0.98(0.89-1.08)$

210

$0.87(0.72-1.05)$

165

$0.94(0.78-1.13)$

$0.66(0.54-0.81)$

$0.75(0.61-0.91)$

$0.84(0.68-1.05)$

$1.04(0.85-1.27)$

1,050

$0.84(0.77-0.91)$

1,044

1.00

1.00

1.00

$0.88(0.81-0.96)$

$0.81(0.74-0.88)$
8,133

$0.64(0.62-0.66)$

$0.78(0.76-0.81)$

$0.84(0.81-0.86)$

1,986

$0.63(0.59-0.66)$

$0.76(0.71-0.80)$

$0.83(0.78-0.88)$

3,391

$0.66(0.63-0.69)$

$0.83(0.79-0.86)$

$0.87(0.83-0.91)$

57

$0.54(0.39-0.74)$

$0.60(0.43-0.83)$

$0.70(0.50-0.99)$

649

$0.52(0.47-0.57)$

$0.80(0.72-0.88)$

$0.89(0.80-0.99)$

3,290

$0.61(0.59-0.64)$

$<0.0001$

$0.80(0.77-0.84)$

$<0.0001$

$0.85(0.81-0.89)$

$<0.0001$

51

$0.45(0.32-0.64) \quad<0.0001$

$0.52(0.37-0.73)$

$<0.0001$

$0.66(0.46-0.94)$

0.005

547

$0.42(0.38-0.46)<0.0001$

$0.70(0.63-0.78) \quad<0.0001$

$0.79(0.71-0.88) \quad<0.0001$

963

157

$0.61(0.50-0.75)$

160

$0.71(0.58-0.87)$

$0.60(0.49-0.74)$

$<0.0001$

$0.71(0.58-0.88) \quad 0.0002$

$0.82(0.66-1.02)$

$0.83(0.67-1.03)$

0.023

Data are hazard ratios (HR) and 95\% confidence interval (Cl). The numbers of deaths are for participants who died during follow-up. The co-variables are baseline assessments.

Model 1, Adjusted for age and gender; Model 2, Adjusted for age, gender, the number of cigarettes smoked per day, and time of smoking cessation $(<1$ years, 1 to 5 years, 5 to 10 years, or $\geq 10$ years before baseline); Model 3, Adjusted for age, gender, the number of cigarettes smoked per day, time of smoking cessation ( $<1$ years, 1 to 5 years, 5 to 10 years, or $\geq 10$ years before baseline), race or ethnicity group, alcohol intake, education level, marital status (yes, no), health status (poor, fair, good, very good), obesity (underweight, overweight, obesity), physical activity, consumption of red meat, total fruit and total vegetables, total energy intake, and hormone usage. 
likely that dietary habits might change during the long (14 years on average) follow-up period, and such temporal patterns were not reflected in our analysis. Moreover, the observational nature of our study limits causality inference between intakes of whole grains or cereal fiber and mortality.

\section{Conclusions}

Data from our study indicate that intake of whole grains and cereal fiber may reduce the risk of all-cause mortality and death from chronic diseases such as cancer, CVD, diabetes, respiratory disease, infections, and other causes. Disappearance or attenuation of whole grain associations with total mortality and death from chronic diseases after adjustment for cereal fiber intake suggests that cereal fiber partly accounts for the protective effects of whole grains on mortality.

\section{Abbreviations \\ Cl: Confidence intervals; CVD: Cardiovascular diseases; FFQ: Food frequency questionnaire; HR: Hazard ratio; NIH: National Institutes of Health.}

\section{Competing interests}

The authors declare that they have no competing interests.

\section{Authors' contributions}

TH and LQ conceived the study. TH, MX, and LQ analyzed the data and wrote the draft of the paper. MX, AL, SC, and LQ contributed to writing, reviewing, and revising of the paper. LQ is the guarantor. All authors read and approved the final manuscript.

\section{Acknowledgments}

We thank all the participants in the NIH-AARP Diet and Health Study. We also thank Dr. David Hasza for statistical assistance.

\section{Sources of support}

This study is funded by an unrestricted research fund from NutraSource. Dr. Qi was supported by grants from the National Heart, Lung, and Blood Institute (HL071981), the National Institute of Diabetes and Digestive and Kidney Diseases (DK091718), the Boston Obesity Nutrition Research Center (DK46200), and United States-Israel Binational Science Foundation Grant 2011036. Dr. Qi was a recipient of the American Heart Association Scientist Development Award (0730094 N). Funding from NutraSource. There were no other relationships or activities that could appear to have influenced the submitted work.

\section{Author details}

'Department of Nutrition, Harvard School of Public Health, 665 Huntington Ave, Boston, MA 02115, USA. ${ }^{2}$ NutraSource (AWL), Royal Oak, Ml 48073, USA. ${ }^{3}$ NutraSource (SSC), Clarksville, MD 21029, USA. ${ }^{4}$ Channing Laboratory, Department of Medicine, Brigham and Women's Hospital and Harvard Medical School, 75 Francis St, Boston, MA 02115, USA.

\section{Received: 29 October 2014 Accepted: 13 February 2015}

\section{Published online: 24 March 2015}

\section{References}

1. Slavin J. Whole grains and human health. Nutr Res Rev. 2004;17:99-110.

2. Qi L, Hu FB. Dietary glycemic load, whole grains, and systemic inflammation in diabetes: the epidemiological evidence. Curr Opin Lipidol. 2007:18:3-8.

3. Qi L, van Dam RM, Liu S, Franz M, Mantzoros C, Hu FB. Whole-grain, bran, and cereal fiber intakes and markers of systemic inflammation in diabetic women. Diabetes Care. 2006;29:207-11.

4. Harland JI, Garton LE. Whole-grain intake as a marker of healthy body weight and adiposity. Public Health Nutr. 2008;11:554-63.
5. de Munter JS, Hu FB, Spiegelman D, Franz M, van Dam RM. Whole grain, bran, and germ intake and risk of type 2 diabetes: a prospective cohort study and systematic review. PLoS Med. 2007;4:e261.

6. Mellen PB, Walsh TF, Herrington DM. Whole grain intake and cardiovascular disease: a meta-analysis. Nutr Metab Cardiovasc Dis. 2008;18:283-90.

7. Aune D, Chan DS, Lau R, Vieira R, Greenwood DC, Kampman E, et al. Dietary fibre, whole grains, and risk of colorectal cancer: systematic review and dose-response meta-analysis of prospective studies. BMJ. 2011;343:d6617.

8. Pajvani UB, Du X, Combs TP, Berg AH, Rajala MW, Schulthess T, et al. Structure-function studies of the adipocyte-secreted hormone Acrp30/ adiponectin: Implications fpr metabolic regulation and bioactivity. J Biol Chem. 2003;278:9073-85.

9. Park Y, Subar AF, Hollenbeck A, Schatzkin A. Dietary fiber intake and mortality in the NIH-AARP diet and health study. Arch Intern Med. 2011;171:1061-8.

10. Adams KF, Schatzkin A, Harris TB, Kipnis V, Mouw T, Ballard-Barbash R, et al. Overweight, obesity, and mortality in a large prospective cohort of persons 50 to 71 years old. N Engl J Med. 2006;355:763-78.

11. Lacey Jr JV, Brinton LA, Leitzmann MF, Mouw T, Hollenbeck A, Schatzkin A, et al. Menopausal hormone therapy and ovarian cancer risk in the National Institutes of Health-AARP Diet and Health Study Cohort. J Natl Cancer Inst. 2006;98:1397-405.

12. Willett W. Nutritional epidemiology: issues and challenges. Int J Epidemiol. 1987;16:312-7.

13. Schatzkin A1, Mouw T, Park Y, Subar AF,Kipnis V, Hollenbeck A, et al. Dietary fiber and whole-grain consumption in relation to colorectal cancer in the NIH-AARP Diet and Health Study. Am J Clin Nutr. 2007;85:1353-60.

14. Thompson FE, Kipnis V, Subar AF, Krebs-Smith SM, Kahle LL, Midthune D, et al. Evaluation of 2 brief instruments and a food-frequency questionnaire to estimate daily number of servings of fruit and vegetables. Am J Clin Nutr. 2000;71:1503-10.

15. International Classification of Diseases, Ninth Revision (ICD-9). World Health Organization. http://www.who.int/classifications/icd/en/

16. International Classification of Diseases (ICD). World Health Organization. Retrieved 23 November 2010. http://apps.who.int/classifications/icd10/ browse/2010/en

17. Willett W. Nutritional epidemiology. 2nd ed. New York: Oxford University Press; 1998.

18. Borneo R, Leon AE. Whole grain cereals: functional components and health benefits. Food Funct. 2012;3:110-9.

19. Slavin J. Why whole grains are protective: biological mechanisms. Proc Nutr Soc. 2003;62:129-34.

20. Ye EQ, Chacko SA, Chou EL, Kugizaki M, Liu S. Greater whole-grain intake is associated with lower risk of type 2 diabetes, cardiovascular disease, and weight gain. J Nutr. 2012;142:1304-13.

21. Flint AJ, Hu FB, Glynn RJ, Jensen MK, Franz M, Sampson L, et al. Whole grains and incident hypertension in men. Am J Clin Nutr. 2009;90:493-8.

22. Egeberg R, Olsen A, Christensen J, Johnsen NF, Loft S, Overvad K, et al. Intake of whole-grain products and risk of prostate cancer among men in the Danish Diet, Cancer and Health cohort study. Cancer Causes Control. 2011;22:1133-9.

23. Schatzkin A, Park Y, Leitzmann MF, Hollenbeck AR, Cross AJ. Prospective study of dietary fiber, whole grain foods, and small intestinal cancer. Gastroenterology. 2008;135:1163-7.

24. He M, van Dam RM, Rimm E, Hu FB, Qi L. Whole-grain, cereal fiber, bran, and germ intake and the risks of all-cause and cardiovascular diseasespecific mortality among women with type 2 diabetes mellitus. Circulation. 2010;121:2162-8.

25. Chuang SC, Norat T, Murphy N, Olsen A, Tjonneland A, Overvad K, et al. Fiber intake and total and cause-specific mortality in the European Prospective Investigation into Cancer and Nutrition cohort. Am J Clin Nutr. 2012;96:164-74.

26. Qi L, Meigs JB, Liu S, Manson JE, Mantzoros C, Hu FB. Dietary fibers and glycemic load, obesity, and plasma adiponectin levels in women with type 2 diabetes. Diabetes Care. 2006;29:1501-5.

27. Esposito K, Giugliano D. Whole-grain intake cools down inflammation. Am J Clin Nutr. 2006:83:1440-1. Author reply 1441-2.

28. Weickert MO, Mohlig M, Schofl C, Arafat AM, Otto B, Viehoff H, et al. Cereal fiber improves whole-body insulin sensitivity in overweight and obese women. Diabetes Care. 2006;29:775-80.

29. Ma Y, Griffith JA, Chasan-Taber L, Olendzki BC, Jackson E, Stanek 3rd EJ, et al. Association between dietary fiber and serum C-reactive protein. Am J Clin Nutr. 2006;83:760-6. 
30. Wannamethee SG, Whincup PH, Thomas MC, Sattar N. Associations between dietary fiber and inflammation, hepatic function, and risk of type 2 diabetes in older men: potential mechanisms for the benefits of fiber on diabetes risk. Diabetes Care. 2009;32:1823-5.

31. Slavin JL. Mechanisms for the impact of whole grain foods on cancer risk. J Am Coll Nutr. 2000;19:300S-7.

32. Jacobs Jr DR, Andersen LF, Blomhoff R. Whole-grain consumption is associated with a reduced risk of noncardiovascular, noncancer death attributed to inflammatory diseases in the lowa Women's Health Study. Am J Clin Nutr. 2007;85:1606-14.

33. Yang J, Martinez I, Walter J, Keshavarzian A, Rose DJ. In vitro characterization of the impact of selected dietary fibers on fecal microbiota composition and short chain fatty acid production. Anaerobe. 2013;23:74-81.

34. Parnell JA, Reimer RA. Prebiotic fibres dose-dependently increase satiety hormones and alter Bacteroidetes and Firmicutes in lean and obese JCR:LA-cp rats. Br J Nutr. 2012;107:601-13.

35. Saura-Calixto F. Dietary fiber as a carrier of dietary antioxidants: an essential physiological function. J Agric Food Chem. 2011;59:43-9.

\section{Submit your next manuscript to BioMed Central and take full advantage of:}

- Convenient online submission

- Thorough peer review

- No space constraints or color figure charges

- Immediate publication on acceptance

- Inclusion in PubMed, CAS, Scopus and Google Scholar

- Research which is freely available for redistribution 